\title{
Chiral symmetry breaking and pair-creation mediated Walker breakdown in magnetic nanotubes
}

Ming Yan, Christian Andreas, Attila Kákay, Felipe García-Sánchez, and Riccardo Hertel

Citation: Appl. Phys. Lett. 100, 252401 (2012); doi: 10.1063/1.4727909

View online: https://doi.org/10.1063/1.4727909

View Table of Contents: http://aip.scitation.org/toc/apl/100/25

Published by the American Institute of Physics

\section{Articles you may be interested in}

Fast domain wall dynamics in magnetic nanotubes: Suppression of Walker breakdown and Cherenkov-like spin wave emission

Applied Physics Letters 99, 122505 (2011); 10.1063/1.3643037

Chirality switching and propagation control of a vortex domain wall in ferromagnetic nanotubes

Applied Physics Letters 100, 072407 (2012); 10.1063/1.3687154

The design and verification of MuMax3

AIP Advances 4, 107133 (2014); 10.1063/1.4899186

Domain wall motion on magnetic nanotubes

Journal of Applied Physics 108, 033917 (2010); 10.1063/1.3466747

Reversal modes in magnetic nanotubes

Applied Physics Letters 90, 102501 (2007); 10.1063/1.2437655

Vortex-antivortex pairs induced by curvature in toroidal nanomagnets

Journal of Applied Physics 121, 113906 (2017); 10.1063/1.4977983

\section{PHYSICS TODAY}

MANAGER'S GUIDE

WHITEPAPERS

\section{READ NOW}

PRESENTED BY

Accelerate R\&D with

Multiphysics Simulation $\checkmark \subset \bigcirc M S L$ 


\title{
Chiral symmetry breaking and pair-creation mediated Walker breakdown in magnetic nanotubes
}

\author{
Ming Yan, ${ }^{1,2, a)}$ Christian Andreas, ${ }^{1,3}$ Attila Kákay, ${ }^{1}$ Felipe García-Sánchez, ${ }^{1}$ \\ and Riccardo Hertel ${ }^{1,3}$ \\ ${ }^{1}$ Peter Grünberg Institut (PGI-6), Forschungszentrum Jülich GmbH, D-52428 Jülich, Germany \\ ${ }^{2}$ Department of Physics, Shanghai University, Shanghai 200444, People's Republic of China \\ ${ }^{3}$ Institut de Physique et Chimie des Matériaux de Strasbourg, Université de Strasbourg, CNRS UMR 7504, \\ Strasbourg, France
}

(Received 27 October 2011; accepted 7 April 2012; published online 18 June 2012)

\begin{abstract}
Field-driven domain wall (DW) propagation in ferromagnetic nanotubes displays unusual effects, as revealed by a micromagnetic study. The left-right symmetry of the DW dynamics is broken, yielding markedly different DW mobilities for opposite propagation directions. This asymmetry arises from the tubular geometry and its impact on the DW structure. Also, the Walker breakdown field and velocity are found to be asymmetric for opposite directions. In certain cases, the breakdown can even be suppressed in one or both directions. Topological constraint requires a vortex-antivortex pair mediated breakdown, contrary to the single (anti)vortex in flat strips. This results in a higher breakdown velocity. (C) 2012 American Institute of Physics. [http://dx.doi.org/10.1063/1.4727909]
\end{abstract}

Recently, the study on magnetic domain wall (DW) dynamics has revived, especially in ferromagnetic nanostructures. Besides its fundamental interest, DW dynamics may play an important role in the emerging field of spintronics. Future devices based on DW displacement, such as the racetrack memory ${ }^{1}$ and magnetic logic gates ${ }^{2}$ have been proposed. For the optimization of such devices, it is essential to use DW guides that support fast and controlled DW motion. Concerning the improvement of the DW speed and stability, the main challenge consists in suppressing the Walker breakdown, ${ }^{3}$ which causes an abrupt change of the DW structure above a critical velocity. The value of the critical velocity is closely related to how the DW alters its structure during the breakdown, which depends sensitively on the geometry of the DW guides. In the original model by Schryer and Walker, Bloch wall propagation in extended films was addressed, and there the DW breakdown resulted from the magnetization precession about the external field. ${ }^{3}$ In laterally confined strips, the breakdown is accompanied by a periodic transformation between transverse and (anti)vortex walls, thus involving the nucleation and annihilation of single (anti)vortices at the lateral boundaries. ${ }^{4}$ For reasons of easy fabrication, magnetic strips are currently the most common choice for DW guides. Theoretical studies, however, suggest that cylindrical wires might have important advantages as potential candidates for DW guides. ${ }^{5-10}$ Nowadays, the feasibility of fabricating round nanowires adds a technological relevance to these studies. ${ }^{11-14}$ In this letter, we describe a complex and previously unreported variant of the Walker breakdown process in cylindrical nanotubes. Due to the absence of lateral boundary, the usual type of breakdown process known from thin strips, which is mediated by the nucleation of a single (anti)vortex, is topologically forbidden

\footnotetext{
${ }^{\text {a) }}$ Author to whom correspondence should be addressed: Electronic mail: m.yan@fz-juelich.de.
}

in nanotubes. ${ }^{15}$ Instead, a more complex process involving a vortex-antivortex pair creation is found, yielding a much higher breakdown threshold. In addition, the curvature of the tube causes a distinctly different effect compared to any flat geometry: the left-right symmetry of the DW propagation is broken. This particular behavior leads to the occurrence of a favorable and unfavorable propagation direction for a given DW. Alternatively, one can identify a favorable and an unfavorable DW chirality for a given propagation direction, which is merely a different perspective on the same effect.

The head-to-head (h2h) or tail-to-tail (t2t) DW structures occurring in flat strips ${ }^{16}$ can be found in a similar way also in round wires, but their configurations differ slightly as they adjust to the geometry. ${ }^{17}$ In particular, a characteristic vortex-like DW can form in hollow wires, i.e., magnetic tubes. ${ }^{18,19}$ The configuration of such a DW is shown in Fig. 1(a). In the region between two oppositely magnetized domains, the magnetization circles around the wire and thereby forms a core-less vortex structure. Such a DW in tubes is in fact analogous to the transverse DW in flat strips, ${ }^{10}$ as illustrated in Fig. 1(b) by artificially "unrolling" the tube. Similar to the two possible orientations of a transverse DW in a strip, this type of DW also occurs in two configurations according to the different vorticity in the DW region. Despite their energetic degeneracy, those two configurations display different dynamic behavior. This is due to the curvature of the tube and its influence on the DW configuration. Unlike transverse DWs in flat strips (shown in Fig. 1(c)), where the magnetization lies perfectly in plane, the magnetization of the DW in a tube has a small but decisive radial component $\left(m_{\rho}\right)$. This is displayed in Fig. 1(b), where $\bar{m}_{\rho}$ (averaged over the cross section of the tube) is plotted as a function of the position $z$. This non-zero $m_{\rho}$ forms in order to reduce one major source of the DW energy, namely the magnetostatic volume charges generated by the DW. By definition, the volume charge density is proportional 

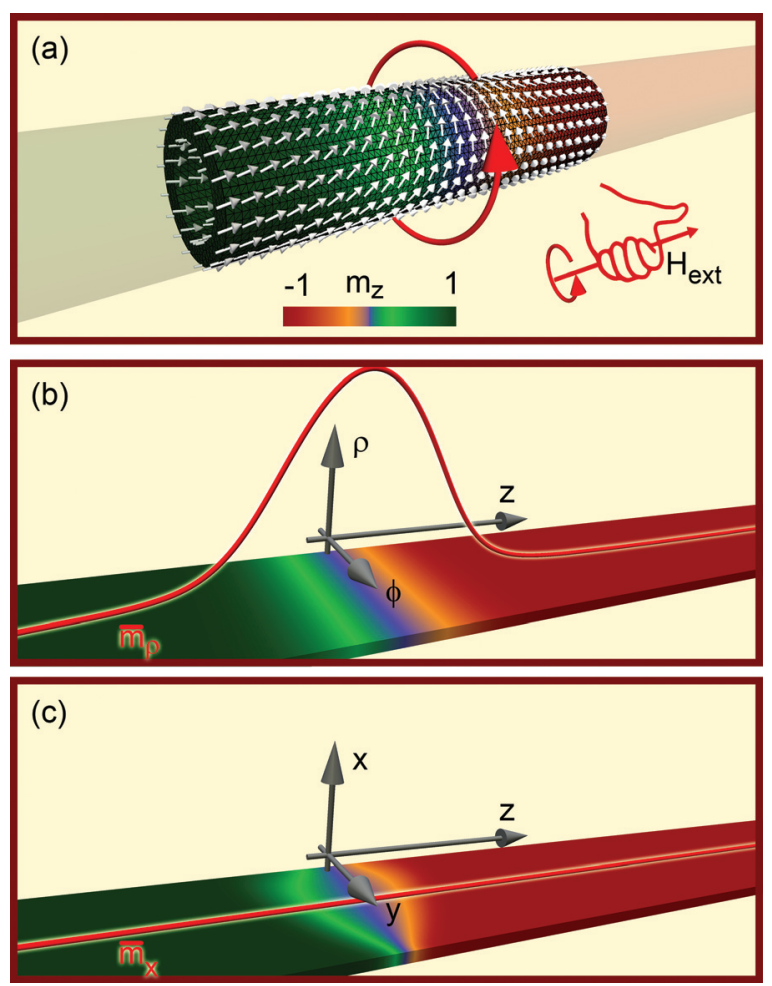

FIG. 1. (a) Simulated configuration of a vortex-like DW formed in a magnetic nanotube and the definition of its chirality by combining vorticity and field direction. (b) The unrolled tube with the DW. The red line is the plot of the radial component averaged over each cross-section. (c) For comparison, a transverse DW in a flat strip.

to the divergence of the magnetization field. In a cylindrical coordinate system shown in Fig. 1(b), the divergence of the magnetization vector $\vec{m}$ reads

$$
\vec{\nabla} \cdot \vec{m}=\frac{m_{\rho}}{\rho}+\frac{\partial m_{\rho}}{\partial \rho}+\frac{1}{\rho} \frac{\partial m_{\phi}}{\partial \phi}+\frac{\partial m_{z}}{\partial z} .
$$

In this DW type, the main contribution of the volume charge density is due to the last term on the right-hand (RH) side of Eq. (1), being negative (positive) for h2h (t2t) DWs. Due to the symmetry of our setup, the third term vanishes. However, the first two terms, which always have the same sign in our calculations, provide a possibility to partially compensate the last term. Therefore, the positive radial component of the h2h DW shown in Fig. 1(b) can be understood as a result of energy minimization. For the same reason, a negative radial component appears in $\mathrm{t} 2 \mathrm{t}$ DWs (not shown). As it will become clear later, this special feature of the DW structure is decisive for the break of left-right symmetry of DW propagation.

The DW shown in Fig. 1(a) can be driven by applying an external field along the tube. The resulting DW propagation is determined by the Landau-Lifshitz-Gilbert (LLG) equation, which is solved numerically:

$$
\frac{d \vec{M}}{d t}=-\gamma \vec{M} \times \vec{H}_{\mathrm{eff}}+\frac{\alpha}{M_{s}}\left[\vec{M} \times \frac{d \vec{M}}{d t}\right],
$$

where $\vec{M}$ is the local magnetization, $M_{s}$ the saturation magnetization, $\gamma$ the gyromagnetic ratio, $\vec{H}_{\text {eff }}$ the effective field, and $\alpha$ the Gilbert damping factor. The calculations are done using our TetraMag finite-element micromagnetic code. ${ }^{20}$ Typical material parameters of Permalloy, $\mu_{0} M_{s}=1.0 \mathrm{~T}$ and exchange constant $A=1.3 \times 10^{-11} \mathrm{~J} / \mathrm{m}$ are used. The sample volume is discretized into irregular tetrahedrons with edge length of about $3 \mathrm{~nm}$. The damping parameter $\alpha$ is fixed to 0.02 .

From the precessional term in the LLG equation it is easy to deduce that the external field exerts a torque on the DW in the radial direction. This torque either increases or reduces the already existing radial component of the static DW, depending on the vorticity of the DW and the field direction. Consequently, a moving DW with a given vorticity is distorted differently, depending on whether it moves to the left or the right. The four possible combinations of DW vorticity and field direction can be categorized according to the relative alignment between the field torque and the initial radial component. A convenient way is to define a chirality of the system by combining the DW vorticity with the field vector, as illustrated in Fig. 1(a). Thereby, the four combinations can be reduced to two cases: left-handed (LH) and RH chirality. The different dynamic behavior of the DW for the two chiralities is displayed in Fig. 2.

The data refer to the case of a h2h DW formed in a $4 \mu \mathrm{m}$ long Permalloy tube with $60 \mathrm{~nm}$ outer diameter and $10 \mathrm{~nm}$ thickness. With a small field of $1 \mathrm{mT}$, the breaking of symmetry is already notable. Figure 2(a) shows the increase and the compression of the radial component of a moving DW in the $\mathrm{LH}$ and in the $\mathrm{RH}$ case, respectively. Remarkably, also the DW mobility (defined as the ratio of the DW velocity and the driving field) shows a strong dependence on the chirality, which is illustrated in Fig. 2(b) by plotting the DW velocity as a function of field in both chirality cases. This plot focuses only on the low-field regime. In the high-field regime, the average DW velocity in the $\mathrm{LH}$ case cannot be defined, as the reason will become clear later. The DW velocity data in the RH case at high fields was presented in an
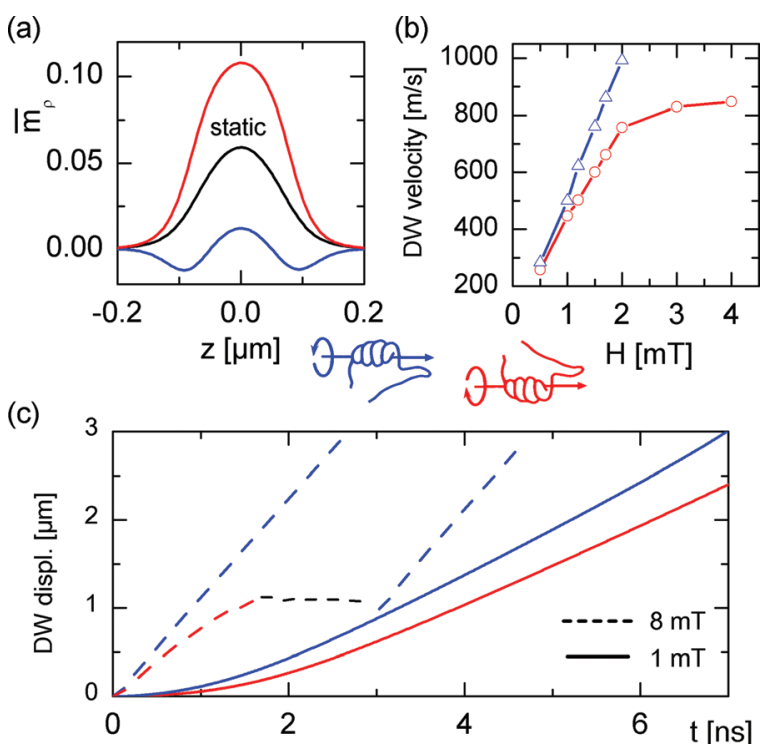

FIG. 2. Comparison of the DW dynamics between the LH chirality case (red) and the RH one (blue). (a) Plot of the averaged radial component $\left(\bar{m}_{\rho}\right)$ of the static DW and two moving DWs. The driving field is $1 \mathrm{mT}$. (b) Average DW velocity as a function of field in the low-field regime. (c) DW displacement as a function of time driven by two different fields. 
early publication ${ }^{10}$ and is not relevant here. Figure 2(c) displays the detailed DW motion driven by a $1 \mathrm{mT}$ field by plotting the DW displacement as a function of time. After a short time of acceleration, the DW reaches a constant speed. The final DW speed in the RH case is clearly higher than the LH one.

The chirality-dependent distortion and mobility of the DW also affect the stability of the DW. The major technological concern about the DW stability is connected with the Walker breakdown, which causes the collapse of the DW structure above a certain critical velocity. The value of this critical velocity usually depends on the material and the geometry of the DW guide. In tubular DW guides, however, we found that this threshold velocity also depends on the DW chirality. In some cases, like the tube discussed here ( $60 \mathrm{~nm}$ outer diameter and $10 \mathrm{~nm}$ thickness), the chiral asymmetry can be maximal in the sense that the Walker breakdown only occurs in the LH chirality case, while in the RH case it is suppressed. This is shown in Fig. 2(c), which displays the DW dynamics in an applied field of $8 \mathrm{mT}$. While in the case of RH chirality the DW moves at a constant speed, it undergoes a conversion process consisting of three stages in the LH case. In the first stage, the DW moves with a relatively small speed compared to the RH chirality case. In the second stage, the DW position remains nearly stationary. In the third stage, the DW resumes its motion and acquires a speed higher than that of the first stage. Notice that the DW speed of the third stage becomes the same as that in the RH chirality case.

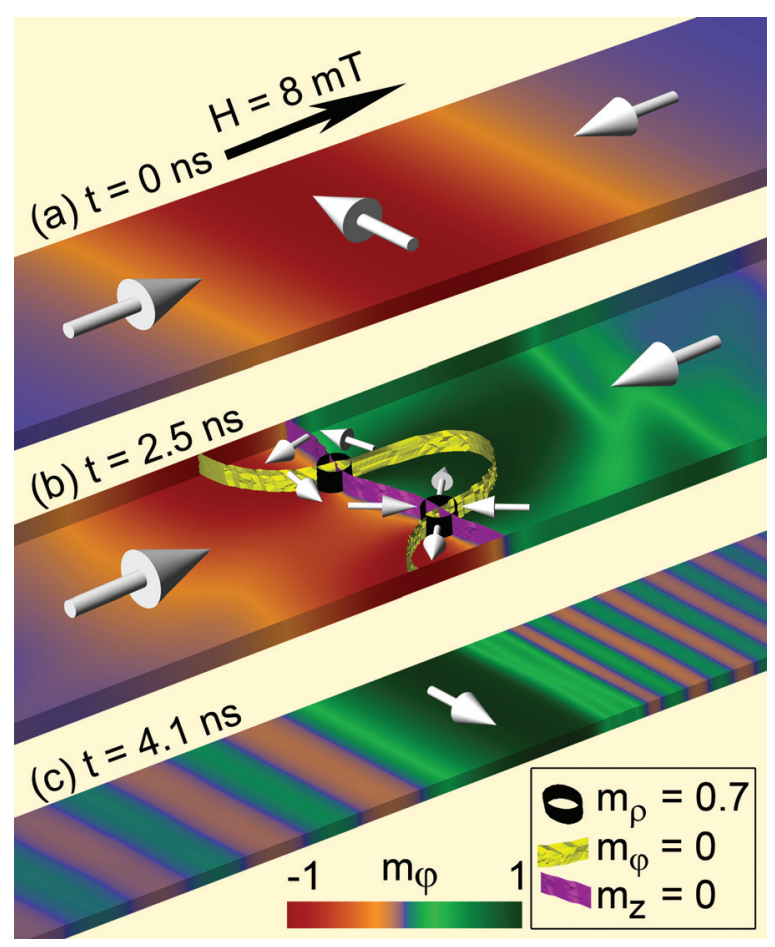

FIG. 3. Snapshots of simulated DW configuration (a) before, (b) during, and (c) after the breakdown process. The magnetic structure is visualized on a flat surface by graphically unrolling the tube onto a strip. The magnetization direction is indicated by the color coding (azimuthal component) and the white arrows. In (b), only the bottom surface and the lateral edges of the strip are shown. Three isosurfaces are utilized to locate the (anti)vortex cores.
This three-staged motion of the DW in the LH chirality case is found to be related to a breakdown process, as shown in Fig. 3. For a better visualization, the unrolled tube is shown. This breakdown process is characterized by the nucleation of a vortex-antivortex pair. In Fig. 3(b), the location of the (anti)vortex core is indicated by the crossing of two isosurfaces $\left(m_{z}=0\right.$ and $\left.m_{\phi}=0\right)$ and isosurface $m_{\rho}=0.7$, respectively. ${ }^{21}$ Once the pair is created, the vortex and the anti-vortex move away from each other. Eventually they meet on the other side of the tube and annihilate. The evolution of this process can be viewed in a supplementary movie. $^{22}$ During the breakdown process, the DW stops moving and may even move backward momentarily, thereby showing a similar velocity drop as the Walker breakdown known from flat strips. Notice that after the annihilation of the pair, the DW vorticity (and hence the chirality) is reversed, as shown in Fig. 3(c). The DW motion switches subsequently to the RH chirality case, as shown in Fig. 2(c). The breakdown process in the tube differs from that in flat strips mainly in two aspects. First, the breakdown in the tube involves a vortex-antivortex pair instead of just a single (anti)vortex as in flat strips. ${ }^{4}$ This is a topological effect resulting from the absence of the lateral boundary in the tubes. The preservation of the winding number requires a vortex-antivortex pair formation in the case of a tube (or an infinitely extended plane), while a nucleation of a single (anti)vortex is permitted in a strip of finite lateral extension. In the case of a tube, the instability of the DW structure sets in when the radial component becomes too large. Due to the topological constraint, this leads to the nucleation of a vortex-antivortex pair. A similar process of vortexantivortex pair creation and annihilation has been well understood in vortex dynamics. ${ }^{23-25}$ Because of the higher energy needed to create a vortex-antivortex pair instead of a single (anti)vortex, the breakdown threshold in tubes is considerably higher than that in flat strips. In this particular case, the breakdown speed is around $800 \mathrm{~m} / \mathrm{s}$, much above the value $(500 \mathrm{~m} / \mathrm{s})$ obtained from a flat strip with the same thickness and a width equal to the circumference of the tube. Secondly, the breakdown occurs only once in the tube and is permanently suppressed afterward, as shown in Fig. 2(c), contrary to the repetitive process in flat strips. This is due to the breaking of the chiral symmetry in tubes, as discussed above.

The Walker breakdown is the ultimate outcome of the DW deformation during its motion. Considering the chiral dependence of the DW distortion, as shown in Fig. 1(a), it is easy to understand that the breakdown threshold depends on the DW chirality, as predicted recently in an analytical model. ${ }^{7}$ In the LH chirality case, the further increase of the radial component favors the nucleation of a vortexantivortex pair, while the opposite happens in the $\mathrm{RH}$ case. Consequently, the breakdown threshold is higher in the $\mathrm{RH}$ chirality case. In fact, the breakdown velocity in the RH case is too high to be reached in this particular case. Instead, the DW encounters a different speed limit, the magnonic limit, ${ }^{10}$ which takes place at a DW velocity superior to the spinwave phase velocity, which is about $1000 \mathrm{~m} / \mathrm{s}$ in this case.

The extent to which the asymmetric effects described in this article influence the DW propagation depends on the 
size of the tubes. Although a full understanding of the size effect remains a topic for future study, it is safe to point out that the chiral asymmetry of the DW mobility is a general feature for all tubes, regardless of their size. This is because it arises from the shape, which gives rise to a qualitative difference between the inner and the outer surface of the tube. Finally, we point out that although only results on h2h DWs are presented, the same physics holds also for $\mathrm{t} 2 \mathrm{t}$ DWs with an opposite chiral preference of the DW mobility.

To conclude from an application point of view, magnetic nanotubes as DW guides have the advantage of high DW stability against the Walker breakdown due to a topological constraint. Given the asymmetric DW mobility, one can expect that a low frequency AC field could lead to a net displacement of the DW. This ratchet-type behavior in nanoscale may also have potential applications. In addition to the DW position, the chirality provides a further degree of freedom for carrying information that can be easily probed by the different mobility.

Note added in proof: A similar study on the switching of domain wall chiralities in nanotubes has been reported recently by J. A. Otálora et al. in an article that was published after the submission of this letter. ${ }^{26}$

This work has been financially supported by the Deutsche Forschungsgemeinschaft through SFB 491.

${ }^{1}$ S. S. P. Parkin, M. Hayashi, and L. Thomas, Science 320, 190 (2008).

${ }^{2}$ D. A. Allwood, G. Xiong, C. C. Faulkner, D. Atkinson, D. Petit, and R. P. Cowburn, Science 309, 1688 (2005).

${ }^{3}$ N. L. Schryer and L. R. Walker, J. Appl. Phys. 45, 5406 (1974).

${ }^{4}$ Y. Nakatani, A. Thiaville, and J. Miltat, Nature Mater. 2, 521 (2003).

${ }^{5}$ M. Yan, A. Kákay, S. Gliga, and R. Hertel, Phys. Rev. Lett. 104, 057201 (2010).
${ }^{6}$ R. Wieser, E. Y. Vedmedenko, P. Weinberger, and R. Wiesendanger, Phys. Rev. B 82, 144430 (2010).

${ }^{7}$ P. Landeros and A. S. Núñez, J. Appl. Phys. 108, 033917 (2010).

${ }^{8}$ Z. Z. Sun, J. Schliemann, P. Yan, and X. R. Wang, Eur. Phys. J. B 79, 449 (2011).

${ }^{9}$ M. Franchin, A. Knittel, M. Albert, D. S. Chernyshenko, T. Fischbacher, A. Prabhakar, and H. Fangohr, Phys. Rev. B 84, 094409 (2011).

${ }^{10}$ M. Yan, C. Andreas, A. Kákay, F. García-Sánchez, and R. Hertel, Appl. Phys. Lett. 99, 122505 (2011).

${ }^{11}$ K. Nielsch, J. Choi, K. Schwirn, R. B. Wehrspohn, and U. Gösele, Nano Lett. 2, 677 (2005).

${ }^{12}$ R. Lv, A. Cao, F. Kang, W. Wang, J. Wei, J. Gu, K. Wang, and D. Wu, J. Phys. Chem. C 111, 11475 (2007).

${ }^{13}$ K. T. Chan, C. Doran, E. G. Shipton, and E. E. Fullerton, IEEE Trans. Magn. 46, 2209 (2010).

${ }^{14}$ K. Pitzschel, J. Bachmann, S. Martens, J. M. Montero-Moreno, J. Kimling, G. Meier, J. Escrig, K. Nielsch, and D. Görlitz, J. Appl. Phys. 109, 033907 (2011).

${ }^{15}$ O. Tchernyshyov and G.-W. Chern, Phys. Rev. Lett. 95, 197204 (2005).

${ }^{16}$ R. D. McMichael, and M. J. Donahue, IEEE Trans. Magn. 33, 4167 (1997).

${ }^{17}$ R. Hertel, J. Magn. Magn. Mater. 249, 251 (2002).

${ }^{18}$ R. Hertel and J. Kirschner, J. Magn. Magn. Mater. 278, L291 (2004).

${ }^{19}$ P. Landeros, O. J. Suarez, A. Cuchillo, and P. Vargas, Phys. Rev. B 79, 024404 (2009).

${ }^{20}$ A. Kákay, E. Westphal, and R. Hertel, IEEE Trans. Magn. 46, 2303 (2010).

${ }^{21}$ This visualization method with isosurfaces has been described in R. Hertel and C. M. Schneider, Phys. Rev. Lett. 97, 177202 (2006).

${ }^{22}$ See supplementary material at http://dx.doi.org/10.1063/1.4727909 for a movie showing the Walker breakdown process.

${ }^{23}$ B. Van Waeyenberge, A. Puzic, H. Stoll, K. W. Chou, T. Tyliszczak, R. Hertel, R. Fähnle, R. Brückl, K. Rott, G. Reiss, I. Neudecker, D. Weiss, C. H. Back, and G. Schütz, Nature (London) 444, 461-464 (2006).

${ }^{24}$ R. Hertel, S. Gliga, M. Fähnle, and C. M. Schneider, Phys. Rev. Lett. 98, 117201 (2007).

${ }^{25}$ K. Y. Guslienko, K.-S. Lee, and S.-K. Kim, Phys. Rev. Lett. 100, 027203 (2008).

${ }^{26}$ J. A. Otálora, J. A. López-López, P. Vargas, and P. Landeros Appl. Phys Lett. 100, 072407 (2012). 